\title{
New Multimedia Teaching Method by Multi - screen Interactive
}

\author{
Panfeng $\mathrm{Li}^{1}$, Jiangshu $\mathrm{Wei}^{1^{*}}$ (corresponding author) \\ ${ }^{1}$ College of Information and Engineering, Sichuan Agricultural University, Ya'an, Sichuan Province, China
}

\begin{abstract}
With the development of electronic technology and the Internet, multimedia has become the main form of class. Under normal circumstances, a computer and a screen can meet the needs of teaching. However, in the case of a large number of students, that might not meet the needs. Therefore, the screen sharing between computer and mobile phone via wireless network is of great importance in multimedia teaching. Nevertheless, the existing routing equipment does not support the broadcast of large streaming video files, so we expect to use a method to reduce the transmission of traffic and make the screen sharing between computer and mobile phone possible.
\end{abstract}

\section{INTRODUCTION}

Screen sharing is not one new concept and in the market, a lot of live software can achieve this function. However, if you want to apply this model in teaching, you will be limited by the cost of the equipment and other restrictions. Due to the particularity of multimedia teaching, we believe that you can abandon the traditional way of full video transmission, and choose the way of script transmission in order to reduce the transmission traffic and solve the hardware constraints!

\section{THE OVERVIEW OF MULTIMEDIA TEACHING}

Multimedia teaching is that in the teaching process, according to the teaching objectives and characteristics of the teaching object, through the teaching design, teachers select and use modern teaching media and traditional teaching methods to participate in the whole process of teaching with students. In this way, teachers can choose a variety of media information for students to form a reasonable structure of teaching process, so as to achieve the optimal teaching effect.

Compared with traditional teaching on blackboard, multimedia teaching uses multi-media computer to comprehensively process and control various media information such as symbols, language, text, sound, graphics, images and video, and organically combine the various elements of multimedia according to the teaching requirements and put them through the screen or projector display. At the same time, multimedia teaching can also add sounds according to the needs, and let the user and the computer have the human-computer interaction to complete the teaching process.
Largely, the use of multimedia teaching methods improves the quality of teaching and the interest of students, therefore, multimedia teaching is the main teaching methods.

\section{SCREEN SHARING TECHNOLOGY}

The screen sharing technology appears after the use of graphical user interface (GUI). The earliest GUI concept was proposed by Apple and quickly adopted by the industry. The purpose of the screen sharing is to reproduce the interface display of the host screen on the remote client. In other words, this approach takes the concept of a virtual terminal so that the remote user feels the same as next to the host. Based on the screen sharing technology, there are many products, such as X-Windows, Windows 2000 remote terminal services, NetMeeting, Symantec's PC Anywhere, Remote Administrator, and the domestic Red Spider teaching system (Liu et al.).

In my view, the screen sharing technology might make multimedia teaching into a new period. In the school computer room, the teacher's demonstration is the application of this technology, so that all student machines can simultaneously display the content of teacher machine. However, in the usual teaching, limited by all kinds of conditions, teachers cannot always use the computer room for teaching. At the same time, mobile phones have become popular products. Therefore, the screen sharing between computer and mobile phone has become a new direction for development (Guo and Liu, 2014). 


\section{WIRELESS TECHNOLOGY}

Wireless network is the use of wireless communication technology. Compared to wired network, wireless network has a greater advantage. Largely, this technology can avoid the limitations of the network. Moreover, the development of wireless network technology also contributes to the development of mobile devices. With the support of the wireless network, mobile devices will be more useful.

At present, the wireless network has become the main form of LAN and the speed of WIFI is being developed from $2.4 \mathrm{G}$ to $5 \mathrm{G}$. Therefore, it is foreseeable that in the future the wireless network has considerable prospects.

\section{SCREEN SHARING BASED ON THE WIRELESS NETWORK}

With the maturity of wireless network technology, the use of wireless network to achieve screen sharing has become a new trend. DLNA technology is the key to make that come true. It solves the interconnection of computers and other electronic products. For example, through wireless or wired networks, the interconnection between mobile phones and flat boards allows us to share our photos, videos and music between the two devices, and makes it possible for unlimited sharing and growth of digital media.

Next, we introduce a piece of software: TeamViewer.

This is a piece of remote control software, and of course, it can be used for the screen sharing, which can be saw in video conference. Nevertheless, TeamViewer is not used in multimedia teaching now. The reason is that it shares the entire screen changes and the data flow is quite large. If hundreds of students use this piece of software at the same time, the router is unable to meet the need so that there will be pause, delay and so on, which should not appear in teaching (Zhang, 2017).

\section{A NEW MULTIMEDIA METHOD BASED ON SCREEN SHARING}

Traditional screen sharing software is real-time recording of the entire screen of the host, and seeds the video to other clients, which can be achieved in the video conference. In our classroom, teachers often use a specific PPT for teaching, so we can transfer this PPT to the student terminals in advance, without the need to transfer the entire screen in real time. When the teacher is teaching, the software only needs to transfer the corresponding script to the student terminal. This greatly reduces the amount of data traffic, and makes it possible for a router to share screen information for multiple devices.

Therefore, in the transmission of scripts, only two aspects need to be considered. One is flip scripts of PPT, and another is the scripts of mouse's virtual brush. For the above two scripts, that is about the getting of the mouse and the keyboard message. We can use the mouse hook and keyboard hook to get those messages. Finally, in the process of date transmitting from the computer to the mobile terminals, only the messages of the keyboard and the mouse are transmitted.

Take the mouse hook for example (Xu, 2006), (Wu and Liu, 2010), (Yuan and Zhang, 2006):

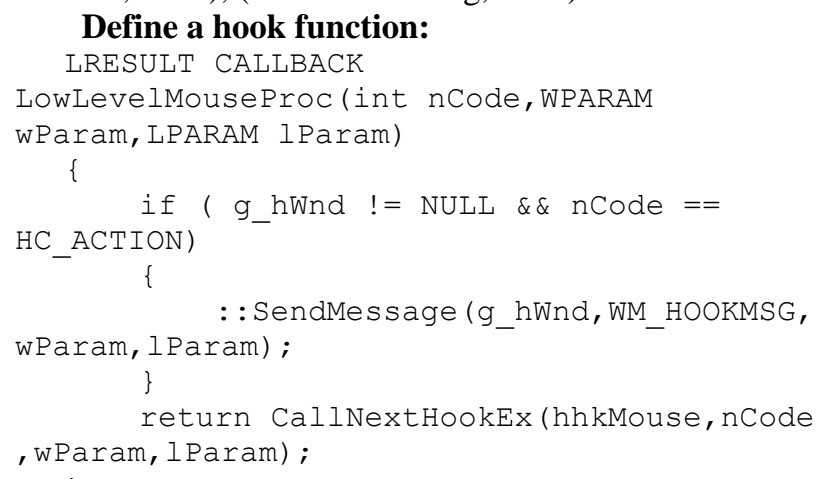

Install the hook:

BOOL WINAPI StartHookMouse (HWND hwnd) \{

g hWnd $=$ hwnd

hh̆kMouse $=$

SetWindowsHookEx (WH MOUSE LL, LowLevelMouse

Proc,g_hInstance, 0);

if ( NULL $==$ hhkMouse)

\}

else

\{

return TRUE;

\}

\}

Uninstall hooks:

VOID WINAPI StopHookMouse()

\{

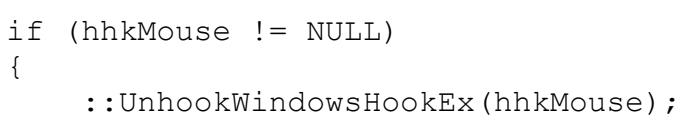

Of course, this is just a low-level hook, and in the application of multimedia teaching, you need to use the global hook, which grabbing the mouse information of the entire screen. Then the message is filtered on the computer side andsent to the mobile terminals through the wireless network. Finally the mobile terminals respond to the corresponding message.

The following is a schematic diagram of the implementation: 


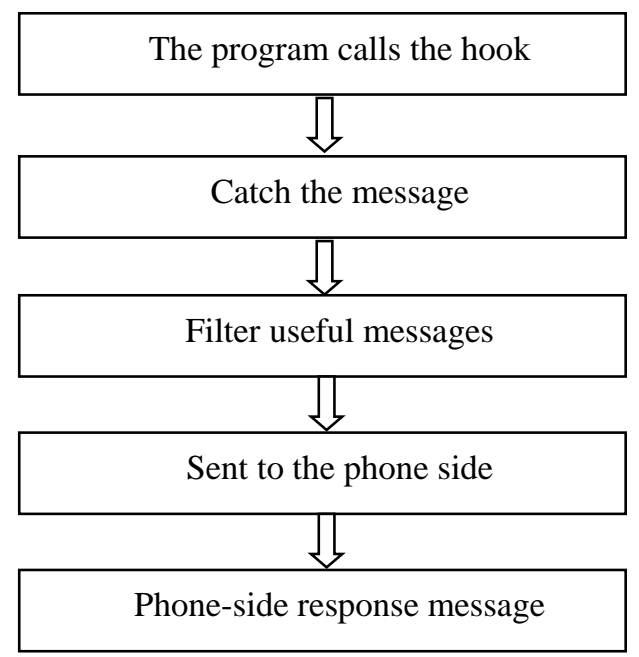

Figure 1: The schematic diagram of the implementation.

\section{SIMULATION EXPERIMENT}

This is an experiment to compare the different performance between transmitting mouse hook scripts and transmitting video data.

Tools: Button Wizard, Cisco Packet Tracer Student.

On a computer with win 10 as the operating system and a resolution of $1920 \times 1080$ screen, the size of a PPT screenshot is about $0.98 \mathrm{MB}$, while the size of the flip scripts of PPT and the scripts of mouse's virtual brush is about $1 \mathrm{~KB}$, which are captured by Button Wizard. The experiment of data transmission in a simulated environment of Cisco network proved that the transmission time of two files is almost proportional to the size of each file.

This is a script captured.

MoveTo 340,331

Delay 1797

LeftDoubleclick 1

Delay 93

Delay 9125

KeyDown "F5", 1

Delay 453

KeyUp "F5", 1

MoveTo 347, 334

Delay 3344

LeftDown 1

LeftClick 1

Leftup 1

Delay 5609

LeftDown 1

LeftClick 1

Leftup 1

Delay 3563

KeyDown "Esc", 1

Delay 218

KeyUp "Esc", 1

MoveTo 1516,146

Delay 7016

LeftDown 1

LeftClick 1

\section{CONCLUSIONS}

Therefore, it is feasible to grab computer operations with mouse hooks, send the messages to mobile terminals, and then let phone-sides respond to the messages. This method improves transmission efficiency. To a large extent, the use of scrip messaging to switch the screen information reduces large data traffic. Although this method has some limitations, it is very meaningful in multimedia teaching.

\section{ACKNOWLEDGEMENTS}

This work is supported by the Project of Innovative Training Program of Sichuan Agricultural University (201610626085).

\section{REFERENCES}

1. Guo Xiaoyan, Liu Cong. Multi-screen Interaction Technology Review. Information Technology \& Standardization, 2014. 9: pp.72-74.

2. Liu Zhenxing, Liu Yang, Tang Shenghong. Outline of Small Internet of Things Multi-Screen Interactive Technology Development. Internet World, 2013.12: pp.13-18.

3. Wu Lin, Liu Ye. Global Keyboard and Mouse Hook Based on VB.NET. Computer Knowledge and Technology, 2010. 36: pp.10328-10331.

4. $\mathrm{Xu}$ Yunjian. The Application of $\mathrm{VC}++$ Hook Mechanism to Windows System. China Science and Technology Information, 2006.13: pp.146-147.

5. Yuan Dongfeng, Zhang Bin. Realization of Global Mouse Hooks under C\#. Computer Programming Skills \& Maintenance, 2006.8: pp. 26-28.

6. Zhang Yan. The Development Trend of Mobile Internet and Multi-screen Interactive Technology. Computer Fan, 2017.7: p.87. 